Check for updates

Cite this: Chem. Commun., 2021, 57,11839

Received 27th September 2021, Accepted 20th October 2021

DOI: $10.1039 / d 1 c c 05455 d$

rsc.li/chemcomm

\section{Di-anionic self-associating supramolecular amphiphiles (SSAs) as antimicrobial agents against MRSA and Escherichia coli†}

\author{
Lisa J. White, ${ }^{a}$ Jessica E. Boles, ${ }^{\text {ab }}$ Melanie Clifford, ${ }^{c}$ Bethany L. Patenall, ${ }^{c}$ \\ Kira H. L. F. Hilton, ${ }^{a}$ Kendrick K. L. Ng, ${ }^{a}$ Rebecca J. Ellaby, ${ }^{a}$ Charlotte K. Hind, ${ }^{* c}$ \\ Daniel P. Mulvihill*b and Jennifer R. Hiscock (D)*a
}

\begin{abstract}
Herein, we report a series of di-anionic supramolecular self-associating amphiphiles (SSAs). We elucidate the antimicrobial properties of these SSAs against both methicillin resistant Staphylococcus aureus and Escherichia coli. In addition, we show this class of compound to form both intra- and intermolecular hydrogen bonded macrocyclic structures in the solid state.
\end{abstract}

Antimicrobial resistance (AMR) is a growing threat to public health worldwide. ${ }^{1}$ The misuse of antibiotics/antimicrobial agents within the veterinarian ${ }^{2}$ and clinical $^{3}$ sectors has contributed to the emergence of multi-drug-resistant organisms, some of which are resistant to all antimicrobial agents currently marketed. ${ }^{4}$ As a result, it has been predicted that by the year 2050 the number of global deaths attributed to the primary effects of AMR will be greater than those caused by cancer (2014), decreasing the worlds GDP by $\$ 100$ trillion. ${ }^{5}$

Supramolecular chemistry has long utilised macrocyclic structures to enable selective host:guest complex formation. ${ }^{6-8}$ Over time these structures have been incorporated into ever more complex systems, which includes but is not limited to; rotaxanes, ${ }^{9}$ catenanes ${ }^{10}$ and biologically inspired molecular machines. ${ }^{11-13}$

While the development of antimicrobial amphiphiles could be considered well known, ${ }^{14-16}$ the exploration of traditional supramolecular macrocyclic scaffolds for the development of novel antimicrobial agents has been of interest since the 1950s, initiated by Macrocyclon. ${ }^{17}$ Naturally occurring peptide based rotaxanes have also been shown to elicit specific bioactivities. ${ }^{18,19}$ For

${ }^{a}$ School of Physical Sciences, University of Kent, Canterbury, Kent, CT2 7NH, UK. E-mail: J.R.Hiscock@Kent.ac.uk

${ }^{b}$ School of Biosciences, University of Kent, Canterbury, Kent, CT2 7NJ, UK.

E-mail:D.P.Mulvihill@Kent.ac.uk

${ }^{c}$ National Infection Service, Public Health England, Porton Down,

Salisbury SP4 0JG, UK. E-mail: charlotte.hind@phe.gov.uk

$\dagger$ Electronic supplementary information (ESI) available: This includes experimental details and DLS, zeta potential, tensiometry, mass spectrometry, NMR spectroscopy, crystallography and microbial data. CCDC 1997690 and 2021377. For ESI and crystallographic data in CIF or other electronic format see DOI: $10.1039 / \mathrm{d} 1 \mathrm{cc} 05455 \mathrm{~d}$ example, microcin J25 acts as an RNA polymerase inhibiter, ${ }^{20}$ a property causing it to be targeted as a potential antimicrobial therapy. ${ }^{21}$ Furthermore, He and co-workers have developed a complex pillar[5]arene which has been shown to effectively carry, deliver and release the antibiotic vancomycin into methicillin resistant Staphylococcus aureus (MRSA), enhancing the intracellular concentration of the antibiotic and thus enhancing efficacy. ${ }^{22}$

Our work in this field to date has led to the identification of a novel class of supramolecular self-associating amphiphiles (SSAs) ${ }^{23,24}$ as: selective phospholipid membrane co-ordination agents, ${ }^{25,26}$ antimicrobial agents against Gram-negative Escherichia coli $\left(E\right.$. coli) and Gram-positive MRSA, ${ }^{27-29}$ anticancer agents, ${ }^{30}$ potential drug delivery vehicles, ${ }^{31}$ and therapeutic efficacy enhancement agents. ${ }^{30,32}$ Here, we present a series of three next-generation di-anionic SSAs (1, 3, 4 - Fig. 1), and report the self-associative properties of these amphiphilic salts

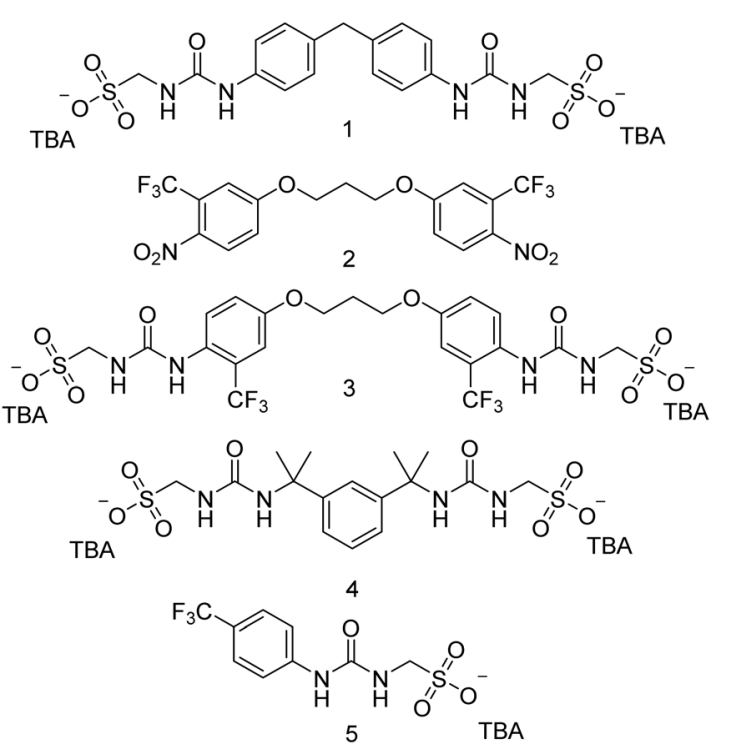

Fig. 1 Chemical structures of di-anionic SSAs (1, 3 and 4), mono-anionic SSA 5 and synthetic intermediate (2). TBA = tetrabutylammonium. 
in the solid state and in aqueous solutions, before exploring the potential of these compounds as antimicrobial agents against both $E$. coli and clinically relevant MRSA.

SSA 1 was obtained through the addition of tetrabutylammonium (TBA) aminomethanesulfonate (AMS) to 4,4'-methylenebis(phenyl isocyanate) and isolated as a cream solid in a yield of $80 \%$. SSA 3 was obtained through the reduction of intermediate 2 , to produce the corresponding amine, which was then reacted with triphosgene to give the corresponding bisisocyanate. This bisisocyanate was then reacted with TBA AMS, to give 3, which was obtained as a yellow solid in a yield of $59 \%$. SSA 4 was obtained through the addition of TBA AMS to 1,3'-bis(1-isocyanato-1-methyl-ethyl) benzene and isolated as a colourless oil in a yield of $67 \%$. The synthesis of SSA 5 has previously been reported. ${ }^{33}$

As observed with the majority of TBA containing monoanionic SSAs, ${ }^{28}$ in the solid state, single-crystal X-ray diffraction studies confirmed the self-association of di-anionic SSAs 1 and 4 through the formation of sulfonate-urea hydrogen bonds. Interestingly, the self-association of $\mathbf{1}$ resulted in the formation of the rectangular macrocycle shown in Fig. 2. Here the formation of 8 intermolecular hydrogen bonds stabilise the formation of this anionic dimer, with two of the four TBA counter cations threading themselves through the anionic macrocycles cavity.

In contrast to $\mathbf{1}$, the anionic component of $\mathbf{4}$ (Fig. 3) selfassociates through the formation of intra-rather than intermolecular sulfonate-anion hydrogen bonds. Although these selfassociative events still result in macrocycle formation, in this instance an anionic, monomeric helical macrocycle is formed, stabilised through the formation of four rather than 8 ureaanion hydrogen bonds.

Moving into the solution state, the presence of any SSA hydrogen bonded self-association events were initially verified through a combination of quantitative ${ }^{1} \mathrm{H}$ NMR, ${ }^{1} \mathrm{H}$ NMR DOSY and ${ }^{1} \mathrm{H}$ NMR dilution studies in a DMSO- $d_{6}$ solution. This is in line with our previous work. ${ }^{23,24,31}$ However, as these data are not the main focus of the work presented here, these data are presented within the ESI $\dagger$ only.
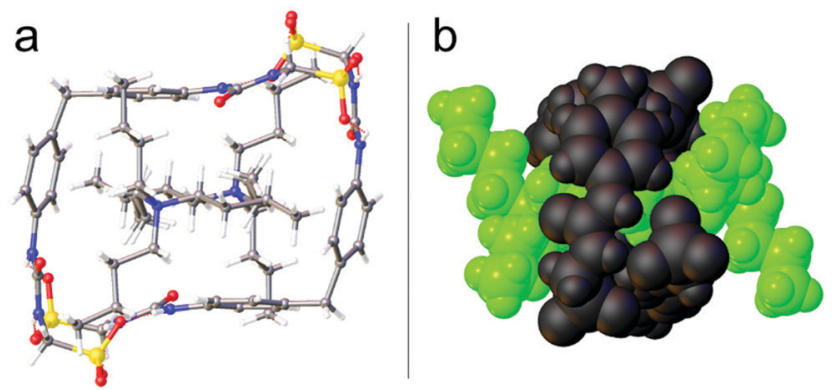

Fig. 2 Single-crystal X-ray structure of 1 (Fig. S46 and Table S6, ESI†) showing the formation of an intermolecular hydrogen bonded macrocycle illustrated as both (a) a ball and stick (grey = carbon, blue = nitrogen, red $=$ oxygen, yellow $=$ sulfur, white $=$ hydrogen, red dashed lines = hydrogen bonds) and, (b) a space filling model (Black = hydrogen bonded, macrocyclic anionic dimer; Green = TBA counter cation). Two of the four TBA counter cations associated with this complex have been omitted for clarity.

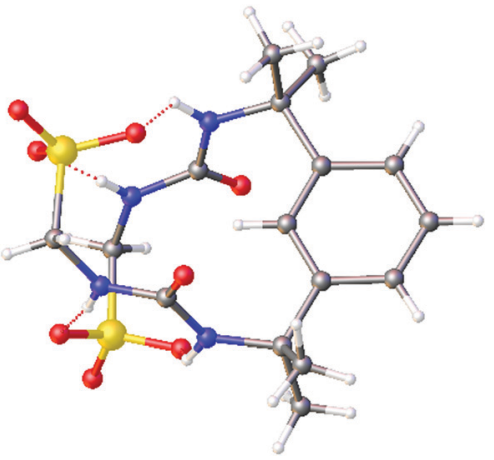

Fig. 3 Single-crystal X-ray structure of 4 (Fig. S47 and Table S7, ESI $†$ ), showing the formation of an anionic, helical macrocycle, stabilised through the formation of four hydrogen bonds. TBA counter cations and associated water molecules have been omitted for clarity. Grey = carbon, blue $=$ nitrogen, red $=$ oxygen, yellow $=$ sulfur, white $=$ hydrogen, red dashed lines $=$ hydrogen bonds.

The self-associative properties of SSAs $\mathbf{1}$ and 3-5 were explored in a $\mathrm{D}_{2} \mathrm{O} / \mathrm{EtOH} 95: 5$ or $\mathrm{H}_{2} \mathrm{O} / \mathrm{EtOH}$ 95: 5 solution, to aid SSA solubility and enable comparison with previously published SSA data. ${ }^{23,24,28}$ Quantitative ${ }^{1} \mathrm{H}$ NMR studies confirmed the presence of aggregated species with solid-like properties at $5.56 \mathrm{mM}$ for all SSAs. The physical properties of these aggregates were elucidated through a combination of dynamic light scattering (DLS), zeta potential and tensiometry (enabling the determination of critical micelle concentration - CMC values) studies (Table 1 ).

Quantitative ${ }^{1} \mathrm{H}$ NMR studies enable the estimation of the proportion of an SSA apparently 'lost' from solution, through the adoption of solid-like properties as a result of higher order self-association events, causing this proportion of molecular species to become NMR silent. Here comparative integration of those signals from the SSA anion or cation against an internal standard (EtOH) allows for the percentage of a molecular component apparently 'lost' from solution to be calculated. When compared to mono-anionic SSA 5, the proportion of di-anionic SSA 1 to become NMR silent under analogous experimental conditions remains comparable. However, this is not the case for $\mathbf{3}$ and $\mathbf{4}$, where not only does the proportion of the SSA to be incorporated into these aggregated structures change, but also the ratios of SSA anion:cation. Here, a 2.10:1.00 and 1.39:1.00 anion:cation ratio is observed for those higher order aggregated structures of 3 and 4 respectively. We hypothesise that in this instance, this is due to partial protonation/deuteration of the SSA's anionic substituent, resulting from solution state aggregation processes. However, this phenomenon remains the subject of ongoing investigations.

The hydrodynamic diameter $\left(d_{\mathrm{H}}\right)$, of those self-associated aggregates produced by SSAs 1, 3, 4 and 5 in a $\mathrm{H}_{2} \mathrm{O} /$ EtOH 95 : 5 solution at $5.56 \mathrm{mM}$ were obtained from DLS studies after undergoing an annealing process ESI. $\dagger$ The stability of those aggregates present within those same solutions was obtained through complimentary zeta potential measurements. The $d_{\mathrm{H}}$ of the aggregates formed by di-anionic SSAs 1, 3 and 4 was found to vary, with $\mathbf{1}$ exhibiting aggregates with the most 
Table 1 Physicochemical data produced to characterise SSA self-association events in a $\mathrm{H}_{2} \mathrm{O} / \mathrm{EtOH}$ 95:5 or $\mathrm{D}_{2} \mathrm{O} / \mathrm{EtOH}$ 95:5 (NMR only) solution. Aggregate stability and $d_{H}$ were obtained via zeta potential and DLS measurements respectively, at a concentration of $5.56 \mathrm{mM}$ and a temperature of $298 \mathrm{~K}$, following an annealing process. The $d_{H}$ of the aggregates listed were obtained from intensity distribution peak maxima. CMC was derived at approximately $291 \mathrm{~K}$ from surface tension measurements. ${ }^{38}$ All quantitative ${ }^{1} \mathrm{H}$ NMR experiments were conducted with a delay time $\left(d_{1}\right)$ of $60 \mathrm{~s}$ at $298 \mathrm{~K}$ and a concentration of $5.56 \mathrm{mM}$. The values given in \% represent the observed proportion of compound to become NMR silent

\begin{tabular}{|c|c|c|c|c|c|c|}
\hline \multirow[b]{2}{*}{ SSA } & \multicolumn{2}{|c|}{${\text { Quantitative }{ }^{1} \mathrm{H} \text { NMR }(\%)^{a}}^{a}$} & \multirow[b]{2}{*}{$d_{\mathrm{H}}(\mathrm{nm})$} & \multirow[b]{2}{*}{ Zeta potential (mV) } & \multirow[b]{2}{*}{$\mathrm{CMC}(\mathrm{mM})$} & \multirow[b]{2}{*}{ Surface tension at $\mathrm{CMC}\left(\mathrm{mN} \mathrm{m}^{-1}\right)$} \\
\hline & anion & cation & & & & \\
\hline 1 & 45 & 52 & 117 & -67 & 24.22 & 49.31 \\
\hline 3 & 82 & 39 & $12,82,5412$ & -74 & 5.77 & 40.76 \\
\hline 4 & 68 & 49 & $1,214,2950$ & -11 & 58.59 & 45.43 \\
\hline $5^{28}$ & 51 & 50 & 164 & -76 & 10.39 & 37.45 \\
\hline
\end{tabular}

${ }^{a}$ Quantitative ${ }^{1} \mathrm{H}$ NMR studies were performed using EtOH as the internal standard.

comparable in size distribution profile to mono-anionic SSA 5. We hypothesise that this may be due to the increased structural rigidity of $\mathbf{1}$ in comparison to $\mathbf{3}$ and $\mathbf{4}$, causing di-anionic SSA $\mathbf{1}$ to adopt aggregate properties that are comparable to monoanionic SSAs. The enhanced flexibility of 3 , afforded through the addition of the central propyl group, combined with the addition of lipophilic, electron withdrawing $\mathrm{CF}_{3}$ substituents, although decreased SSA aggregate uniformity, was found to improve/maintain aggregate stability when compared to $\mathbf{1}$ and 5 respectively. However, both aggregate uniformity and stability was decreased with $\mathbf{4}$, conceivably due to competitive intramolecular hydrogen bond formation events.

When ranking this group of SSAs according to CMC value, we observe the following: $\mathbf{4}>\mathbf{1}>\mathbf{5}>\mathbf{3}$ (Table 1). Here, in line with previous observations, ${ }^{28}$ SSA anion lipophobicity and intermolecular hydrogen-bond formation events are hypothesised to influence critical micelle concentration (CMC) values. With the addition of the $\mathrm{CF}_{3}$ and propyl linker groups found to lower the CMC of di-anionic SSA 3 below that obtained for mono-anionic SSA 5.

Within the scope of our antimicrobial efficacy studies, the SSA is added to the microbial culture in a $\mathrm{H}_{2} \mathrm{O} / \mathrm{EtOH}$ 95:5 solution. This ensures that the SSA antimicrobial effects can be directly related to those SSA aggregates characterised in Table 1 . We have previously shown mono-anionic SSAs to interact with, and permeate bacterial membranes. ${ }^{25,28,30,32}$ The results summarised in Table 2 show the di-anionic SSAs have an enhanced antimicrobial selectivity for Gram +ve MRSA over Gram -ve E. coli and a low cytotoxicity profile against human red blood cells. Interestingly, when ranking the \%

Table $2 \mathrm{MIC}_{50}$ values ( $\mathrm{mM}$ ) determined for 1, 3, 4 and 5 against clinically relevant Gram +ve MRSA USA300 and Gram -ve E. coli DH1OB at an initial calibrated cell concentration equal to the $0.5 \mathrm{McF}$ arland standard, after 900 mins. The cytotoxicity of the SSAs was measured by haemolysis of human red blood cells at $2 \mathrm{mM}$ of SSA. ${ }^{35}$ Error $=$ standard deviation to $2 \mathrm{dp}$

\begin{tabular}{lllr}
\hline SSA & MRSA & E.coli & \% Haemolysis \\
\hline $\mathbf{1}$ & $1.29 \pm 0.15$ & $a$ & $8.2 \pm 3.66$ \\
$\mathbf{3}$ & $0.44 \pm 0.04$ & $a$ & $13.0 \pm 0.44$ \\
$\mathbf{4}$ & $0.91 \pm 0.04$ & $22.5 \pm 0.02$ & $0.7 \pm 0.09$ \\
$\mathbf{5}^{32}$ & $0.46 \pm 0.03$ & $3.85 \pm 0.07$ & $6.3 \pm 2.23$
\end{tabular}

${ }^{a}$ SSA did not pass initial antimicrobial screening, exhibited $<10 \%$ inhibition of growth at $3.3 \mathrm{mM}$ after 900 mins ESI. haemolysis induced by $\mathbf{1}, \mathbf{3 - 5}$, this was found to differ from the ranking of antimicrobial activity. Furthermore, di-anionic SSA 4 was found to exhibit a $\%$ haemolysis 9 times less than the mono-anionic SSA 5. These observations act as evidence towards the hypothesis that SSA technology can be tailored with bespoke activity towards different cell lines, thus enabling the control of unwanted toxic effects, and highlighting the potential for this technology for further development into the clinic.

The antimicrobial efficacy of di-anionic SSAs 1, 3 and $\mathbf{4}$ was found to decrease with increasing \% of the SSAs anionic component to be incorporated into solid-like aggregated structures (Table 1 - quantitative ${ }^{1} \mathrm{H}$ NMR). This causes us to hypothesise that the di-anionic SSA mode of action maybe dependent on a critical concentration of SSA to arrive at the microbial surface in an aggregated form before an antimicrobial effect can be achieved, ${ }^{24,27,28,32}$ in an analogous fashion to that described by the antimicrobial carpet mechanism. ${ }^{34}$ However, it is also conceivable that the flexibility and lipophobicity of the di-anion central linker may be a contributing factor to aid membrane permeation, through effective shielding of the SSA anions charged functionalities.

In conclusion, we report a series of next-generation, dianionic SSAs and characterise the self-associative properties of these amphiphilic salts within the solution state ESI $\dagger$ and solid state. We show how intra- or intermolecular SSA di-anion hydrogen bond formation maybe used to produce new classes of self-associated macrocycles, highlighting the potential of this structural motif to be developed as foldamers, rotaxanes or catenanes. In addition, we report the enhanced antimicrobial selectivity of di-anionic SSAs for clinically relevant Gram +ve MRSA over Gram - ve E. coli and show these agents to exhibit low toxicity towards human red blood cells. It is the combination of these data that highlights the potential of this technology to not only be developed into the clinical space, but also for the production of novel classes of complex, selfassembled supramolecular systems. As a result of these findings we now aim to focus our work to derive the mechanism of antimicrobial action for this class of agents.

L. White: investigation; validation; writing - original draft. J. Boles, B. Patenall and M. Clifford: investigation; writing original draft. K. Hilton, $\mathrm{K}$. $\mathrm{Ng}$ and R. Ellaby: investigation. C. Hind and D. Mulvihill: funding acquisition; validation; 
supervision; writing - review \& editing. J. Hiscock: conceptualization; funding acquisition; project administration; supervision; writing - original draft, review \& editing.

J. H. and L. W. would like to thank the UKRI for JH's Future Leaders Fellowship (MR/T020415/1). J. B. would like to thank Public Health England and the University of Kent for PhD studentship funding.

\section{Conflicts of interest}

There are no conflicts to declare.

\section{References}

1 B. Aslam, W. Wang, M. I. Arshad, M. Khurshid, S. Muzammil, M. H. Rasool, M. A. Nisar, R. F. Alvi, M. A. Aslam, M. U. Qamar, M. K. F. Salamat and Z. Baloch, Infect. Drug Resist., 2018, 11, $1645-1658$.

2 S. J. Bright-Ponte, Zoonoses Public Health, 2020, 67, 1-5.

3 E. D. Brown and G. D. Wright, Nature, 2016, 529, 336-343.

4 P. Davey, C. A. Marwick, C. L. Scott, E. Charani, K. McNeil, E. Brown, I. M. Gould, C. R. Ramsay and S. Michie, Cochrane Database Syst. Rev., 2017, 1-315.

5 S. Davies, J. Farrar, J. Rex, L. White, R. Murry and J. O'Neill, Antimicrobial Resistance: Tackling $a$ crisis for the health and wealth of nations, 2014.

6 X. Wu, A. M. Gilchrist and P. A. Gale, Chem, 2020, 6, 1296-1309.

7 J. R. Nitschke, Angew. Chem., 2017, 129, 39.

8 S. N. Berry, L. Qin, W. Lewis and K. A. Jolliffe, Chem. Sci., 2020, 11, 7015-7022.

9 M. Xue, Y. Yang, X. Chi, X. Yan and F. Huang, Chem. Rev., 2015, 115, 7398-7501.

10 J. D. Crowley, S. M. Goldup, A.-L. Lee, D. A. Leigh and R. T. McBurney, Chem. Soc. Rev., 2009, 38, 1530-1541.

11 L. M. Ceo and J. T. Koh, ChemBioChem, 2012, 13, 511-513.

12 X. Tang, J. Zhang, J. Sun, Y. Wang, J. Wu and L. Zhang, Org. Biomol. Chem., 2013, 11, 7814.

13 A. Acevedo-Jake, A. T. Ball, M. Galli, M. Kukwikila, M. Denis, D. G. Singleton, A. Tavassoli and S. M. Goldup, J. Am. Chem. Soc., 2020, 142, 5985-5990.

14 N. A. Falk, J. Surfactants Deterg., 2019, 22, 1119-1127.

15 A. Pinazo, M. A. Manresa, A. M. Marques, M. Bustelo, M. J. Espuny and L. Pérez, Adv. Colloid Interface Sci., 2016, 228, 17-39.

16 T. Hamouda and J. R. Baker, J. Appl. Microbiol., 2000, 89, 397-403.

17 J. W. Cornforth, P. D. Hart, G. A. Nicholls, R. J. W. Rees and J. A. Stock, Br. J. Pharmacol. Chemother., 1955, 10, 73.
18 K. J. Rosengren, A. Blond, C. Afonso, J.-C. Tabet, S. Rebuffat and D. J. Craik, Biochemistry, 2004, 43, 4696-4702.

19 A. Moretto, M. Crisma, F. Formaggio and C. Toniolo, Biomol. Concepts, 2012, 3, 183-192.

20 K.-A. Wilson, M. Kalkum, J. Ottesen, J. Yuzenkova, B. T. Chait, R. Landick, T. Muir, K. Severinov and S. A. Darst, J. Am. Chem. Soc., 2003, 125, 12475-12483.

21 J. Mukhopadhyay, E. Sineva, J. Knight, R. M. Levy and R. H. Ebright, Mol. Cell, 2004, 14, 739-751.

22 H. Peng, B. Xie, X. Yang, J. Dai, G. Wei and Y. He, Chem. Commun., 2020, 56, 8115-8118.

23 L. J. White, S. N. Tyuleva, B. Wilson, H. J. Shepherd, K. K. L. Ng, S. J. Holder, E. R. Clark and J. R. Hiscock, Chem. - Eur. J., 2018, 24, 7761-7773.

24 L. J. White, N. J. Wells, L. R. Blackholly, H. J. Shepherd, B. Wilson, G. P. Bustone, T. J. Runacres and J. R. Hiscock, Chem. Sci., 2017, 8, 7620-7630.

25 G. Townshend, G. S. Thompson, L. J. White, J. R. Hiscock and J. L. Ortega-Roldan, Chem. Commun., 2020, 56, 4015-4018.

26 E. Medina-Carmona, L. Varela, A. C. Hendry, G. S. Thompson, L. J. White, J. E. Boles, J. R. Hiscock and J. L. Ortega-Roldan, Chem. Commun., 2020, 56, 11665-11668.

27 S. N. Tyuleva, N. Allen, L. J. White, A. Pépés, H. J. Shepherd, P. J. Saines, R. J. Ellaby, D. P. Mulvihill and J. R. Hiscock, Chem. Commun., 2019, 55, 95-98.

28 N. Allen, L. J. White, J. E. Boles, G. T. Williams, D. F. Chu, R. J. Ellaby, H. J. Shepherd, K. K. L. Ng, L. R. Blackholly, B. Wilson, D. P. Mulvihill and J. R. Hiscock, ChemMedChem, 2020, 15, 2193-2205.

29 L. J. White, J. E. Boles, N. Allen, L. S. Alesbrook, J. M. Sutton, C. K. Hind, K. L. F. Hilton, L. R. Blackholly, R. J. Ellaby, G. T. Williams, D. P. Mulvihill and J. R. Hiscock, J. Mater. Chem. $B, 2020,8,4694-4700$.

30 N. O. Dora, E. Blackburn, J. E. Boles, G. T. Williams, L. J. White, S. E. G. Turner, J. D. Hothersall, T. Askwith, J. A. Doolan, D. P. Mulvihill, M. D. Garrett and J. R. Hiscock, RSC Adv., 2021, 11, 14213-14217.

31 L. J. White, J. E. Boles, K. L. F. Hilton, R. J. Ellaby and J. R. Hiscock, Molecules, 2020, 25, 4126.

32 J. E. Boles, R. J. Ellaby, H. J. Shepherd and J. R. Hiscock, RSC Adv., 2021, 11, 9550-9556.

33 J. R. Hiscock, G. P. Bustone, B. Wilson, K. E. Belsey and L. R. Blackholly, Soft Matter, 2016, 12, 4221-4228.

34 J. A. F. Corrêa, A. G. Evangelista, T. de, M. Nazareth and F. B. Luciano, Materialia, 2019, 8, 100494.

35 S. M. Travis, N. N. Anderson, W. R. Forsyth, C. Espiritu, B. D. Conway, E. P. Greenberg, P. B. McCray, R. I. Lehrer, M. J. Welsh and B. F. Tack, Infect. Immun., 2000, 68, 2748-2755. 